\title{
Clinical Aspect of Kidney Diseases
}

\author{
Renal Circulation* \\ Jugoro TAKEUCHI \\ The First Department of Internal Medicine, School of Medicine, \\ Kanazawa University
}

Until recently, not enough attention has been payed to renal circulation because its disturbances seldom threaten a life immediately in comparison with those of cerebral or coronary circulation. However, modern development in the field of renal physiology especially on the mechanism of autoregulation of renal circulation and on the medullary function based on counter-current theory has increasingly forced us to be interested in renal, especially intrarenal circulation.

In addition, it is well recognized clinically that the vascular lesions of the kidney are observed not only in the diseases of the kidney itself, but also in all kinds of hypertensive disease and in those which cause generalized vascular lesions, such as collagen disease or diabetes mellitus. It has also been known that functional or organic disorders of the renal vasculature may actively contribute to the progression of renal diseases.

In this report, I would like to make comments on renal circulation from the standpoint of morphology, physiology and pathophysiology.

\section{The Vasculature of the Kidney}

Mode of branching of the segmental arteries in the kidney: The mode of branching is classified into 4 types: in type $\mathrm{A}$, the main renal artery divides into the anterior and posterior branches; in type B, either the upper or the lower segmental artery branches out first; in type $C$, more than 3 branches come from the renal artery at the same time; type D is "multiple renal arteries". The incidence of multiple renal arteries was $21 \%$. It was Graves" ${ }^{12}(1954)$ who established the concept of the segment in human kidney. He divided the kidney into 5 segments but we think it is more rational to divide it into 4 segments, which are supplied blood from each segmental (interlobar) artery.

Arteriole-glomerular units and juxtamedullary circulation: It has been clearly shown by Zlábek ${ }^{2)}$, Eguchi ${ }^{37}$ and Ljungqvist ${ }^{4)}$ that there are two types of arterioleglomerular units in the human kidney. In type I, all blood flows through the glo-

* Presented at the 65 th Annual Meeting of the Japanese Society of Internal Medicine, April 2, 1968, in Tokyo. 
merular tuft, while in type II, part of the blood by-passes the glomerular tuft. Most of the cortical glomeruli belong to type I and we suppose that the majority of juxta. medullary glomeruli belong to type II.

In the cortex, arteriole-glomerular units disappear in association with deterioration of glomeruli, while in juxta-medullary area, continuity between the afferent and the efferent arterioles becomes distinct in degeneration of glomeruli and the ratio of thus developed arteriolae rectae verae to total arteriolae rectae increases with age. The ratios in chronic glomerulonephritis and nephrosclerosis were increased beyond the increment of the ratio with age.

Medullary circulation: The renal medulla received blood from the ordinary arteriolae rectae, the giant afferent arterioles (Ljungqvist) and its incomplete form, and the spiral arteries. These vessels arise from different parts of the renal vasculature, but appear to have a similar physiological function. In nephrosclerosis and contracted kidney due to chronic glomerulonephritis, relatively well preserved medullary vessels with increased vasae rectae verae were observed, in spite of an extensive involvement in the cortical vasculature.

\section{Separate Measurement of Cortical and Medullary Circulation}

For this purpose, we made an attempt to devise a new analytical method of dye-dilution curves. Indocyanine green was directly injected into one renal artery through a catheter. Dye-dilution curves were recorded by constant withdrawing of blood through ipsilateral renal vein catheter, using a densitometer. Then, a venous catheter only was inserted in another renal vein and recirculation curves were recorded which were subtracted from the original curves. For analyzing dye-dilution curves, we supposed that a curve was made of two components, a fast one (cortical) and a slow one (medullary), and that the passage time of dye in the cortex and medulla had a log-normal distribution respectively. Dye-dilution curve will be expressed in the following formula;

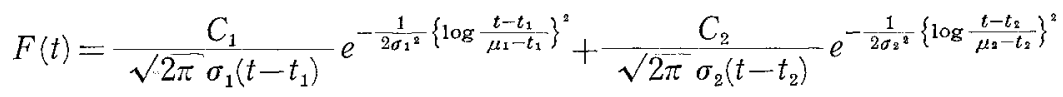

where $C_{1}, C_{2}$ represent the area under cortical and medullary components (curves) respectively, $\sigma_{1}, \sigma_{2}$ : width of each curve, $t_{1}, t_{2}$ : appearance time of each curve and $\mu_{1}, \mu_{2}:$ median value of each curve.

Curve fittings were performed by the least square method, utilizing a digital computer. Here, we supposed that the appearance of medullary component comes after the peak of dye-dilution curve.

Eighteen patients with hypertension or renal parenchymal diseases were selected for a study and 32 curves from these patients were analyzed. The cortical flow was significantly decreased in 11 azotemics as compared with 7 normal subjects, while the difference of the changes in medullary flow was insignificant between two groups. 
Therefore, the ratio of the medullary flow to the total flow was larger in azotemics than in normal cases. These findings are consistent with the above mentioned findings on the renal vasculature in azotemics.

This new analytical method has following advantages; the entire curve, not merely the descending limb as in graphical method, is evaluated mathematically and the influence of recirculation is eliminated.

\section{Morphological Observations on Renal Circulation-Renal Angiography}

Aortography and selective renal angiography are mainly used in diagnosis of renovascular hypertension. These methods are also of considerable value in observing the intrarenal hemodynamics.

Essential hypertension and multiple renal arteries: Higher incidence of multiple renal arteries was reported by several authors and they supposed that some microscopic or submicroscopic disturbance of intrarenal blood flow accompanied with multiple renal arteries may contribute to the genesis of hypertension ${ }^{52}$. In our observation, the incidence of multiple renal arteries in essential hypertension was 1.65 times as high as in non-hypertensives but the difference was not statistically significant at $5 \%$ level.

Differential diagnosis between essential hypertension and chronic glomerulonephritis: In essential hypertension, the angle of the first branchings is, angiographically, wider and the arteries become narrower rather suddenly in peripheral parts and look like dead tree branches. On the contrary, in chronic glomerulonephritis, the renal artery becomes narrower from the beginning of the main artery as the kidney deteriorates in its function.

\section{Regulation of Renal Circulation}

Renal circulation is regulated by extrarenal (nervous and humoral) and intrarenal (autoregulatory) mechanisms.

Nervous control of the renal circulation: Morphologically it is clearly shown that the renal vasculature received abundant nerve supply and that functionally it is regulated by the higher autonomic centers and reflex via baroreceptors.

Higher autonomic centers and renal circulation: It is a well known fact that under stress or in an emergency state, renal blood flow decreases markedly. Our experiment involving electrical stimulation of the diencephalon in dogs demonstrated that there were renal vasoconstricting areas in most of the hypothalamic and subthalamic nuclei and in the medial and midline nuclei group in the thalamus ${ }^{6}$.

Baroreceptors and renal circulation: With an increase in pressure to the baroreceptors, action potentials of the efferent renal nerve decreased and they increased with a decrease in pressure to the baroreceptors. However, reflex action potentials of the renal nerve via the baroreceptors is supposed to be affected by sometimes 
cooperative and, in other times, antagonistic actions from the higher autonomic centers. The interactions between the baroreceptors and the higher centers remain to be studied.

Humoral control: Stimulation of the sympathetic nerve results in a rise of cathecholamine and renin-angiotensin in the blood which further causes renal vasoconstriction. Humoral control mechanism together with nervous control mechanism compose the neuro-humoral control mechanism on renal circulation.

Autoregulation: When changing arterial perfusion pressure to the kidney within a certain range, renal blood flow tends to remain constant. Denervation or elimination of humoral factors does not affect this phenomenon which is called autoregulation of renal circulation. Although the mechanism of renal autoregulation is not fully understood, we suppose that it is mainly based on the nature of the renal vasculature itself (myogenic theory).

The autoregulatory mechanism is abolished not only in paralysis of the vessels caused by papaverine hydrochloride but also in vasoconstriction induced by epinephrine, norepinephrine or angiotensin.

\section{Clinical Aspects of Renal Vascular Lesions from the Standpoint of Renal Circulation}

Functional disorders of the renal vasculature: Acute cortical necrosis and acute tubular necrosis (ATN) are the major diseases in this field. I would like to discuss only the latter, because the former is quite rare.

Specimens of the kidney with ATN were examined in 2 biopsy cases in our department and 8 autopsy cases from the Department of Pathology, Kanazawa University. Major findings were dilated tubules with flattened epithelial cells, degeneration and necrosis of tubular cells, interstitial edema with minor glomerular or vascular changes. The mechanism of oliguria in ATN has been postulated by following sequence of events; ischemia and/or nephrotoxic substance-renal tubular damage-back diffusion of intraluminar fluid to the interstitium-increased intrarenal pressure-mechanical obstruction of renal blood flow-decreased GFR-oliguria. However, the discrepancy between the degree of interstitial edema and oliguria, and the abrupt onset of diuresis in the course of ATN rather suggest the possibility: that some kind of changes in intrarenal circulation such as constriction of the afferent arterioles is responsible for a decreased GFR.

Organic disorders of the renal vasculature:

a. Lesion in the renal arterial system.

Lesions in the main renal artery and its large. branches: Clinically, the most important problem in this field is renovascular hypertension. According to the answers to our questionaire, the most frequent cause of this disease was aortitis syndrome in young females (66 out of 219 patients) in this country, as already reported by Ueda. 
Lesions of the intrarenal arteries: Aging process of the small artery and the arterioles in the kidney was studied in autopsy specimens from patients without hypertensive or generalized vascular diseases. Intimal thickening of small arteries started from early in the 3rd decade and arteriolosclerosis appeared in the 5 th decade. Hypertension accelerates both arterio- and arteriolosclerosis, especially the latter. The etiology of fibrinoid necrosis in malignant nephrosclerosis is a matter of controversy. Some authors attach importance to marked diastolic hypertension and others suppose the existence of some vascular injuring factors. Our study of the relationship between the grade of arteriolosclerosis and the diastolic blood pressure showed that only high levels of diastolic pressure (above $120 \mathrm{~mm}$. $\mathrm{Hg}$ ) is associated with fibrinoid necrosis.

It is conceivable that the marked vascular lesions in the kidney affect renal circulation. The relation of the degree of the vascular lesions to renal clearances $\left(\mathrm{C}_{\mathrm{PAH}}\right.$ and $\mathrm{C}_{\text {Thiosulfate) }}$ was examined. Those with malignant nephrosclerosis showed marked decrease in renal clearances; benign nephrosclerosis itself seldom causes such a marked functional impairment but some of them have GFR of 50 to $60 \mathrm{ml}$./ $\mathrm{min}$. and $\mathrm{RPF}$ of 250 to $300 \mathrm{ml} . / \mathrm{min}$. and it is worthwhile noting that in these cases moderate dehydration or hypotension may precipitate renal failure.

Diabetes mellitus is considered as another factor which accelerates the arteriolosclerosis of the kidney. The longer the duration of diabetes mellitus was, the more severe was the degree of arteriolosclerosis. The characteristics of diabetic arteriolosclerosis is that hyalinization occurs not only in the afferent but also in the efferent arteriole. This does not make us regard diabetic arteriolosclerosis as a mere acceleration of an aging process.

b. Glomerular lesions and renal circulation

We have studied the relationship between glomerular lesions and renal circulation in chronic glomerulonephritis, membranous glomerulopathy, and diabetic glomerulosclerosis.

Glomerular lesion curves: To express the degree and homogenicity of glomerular lesion, a glomerular lesion curve drawn in the following way was used. First, glomerular lesions were classified into 5 grades, grade 0 , no change to grade IV, almost or completely hyalinized. Frequencies (percentages) of each glomerular lesion were plotted against the grade and connected by line to each biopsy or autopsy specimen. In evaluating the glomerular lesion curve of a group (e.g. a group with a decreased renal function), all glomeruli in the specimen belonging to the group were accumulated. In this case, 15 representative glomeruli (average number of glomeruli in biopsy specimens) were selected from autopsy specimens for evaluation.

Chronic glomerulonephritis: One hundred and seven cases of chronic glomerulonephritis with mesangial change and without nephrotic syndrome were divided into 3 groups by the degree of renal function; normal or subnormal (GFR $\geqq 80, R P F \geqq 400$ ), slightly decreased $(80>\mathrm{GFR} \geqq 50,400>\mathrm{RPF} \geqq 250)$ and markedly impaired group $(50>$ GFR, 250>RPF). 
Glomeruli consist mainly of grade I lesions in the normal function group and grade IV lesions, in the highly impaired function group. The group with slightly decreased function showed intermediate types between the other two groups. Looking at each case, there are two characteristics of chronic glomerulonephritis; a broad distribution of glomerular lesions and rarity of cases which consist mainly of grade III lesion. This suggests that once glomerular lesion reaches grade IIl, it tends to progress rapidly to grade IV (hyalinization).

Seventeen cases of nephrotic syndrome with mesangial change showed a similar tendency, but those with poor function showed less frequency of grade IV glomeruli suggesting the necessity of functioning glomeruli in the development of nephrotic syndrome.

Membranous glomerulopathy: Seventeen cases with diffuse thickening of glomerular basement membrane under light microscope which are thought to be membranous glomerulopathy, were studied. This disease is characterized by a relatively homogenous glomerular lesion and it seems to us that before lesions of the glomeruli progress to grade IV change, patients are entering into terminal renal failure.

Diabetic glomerulosclerosis: Since diffuse glomerular lesion affects renal circulation more than nodular lesion does, 55 cases with diffuse lesion were evaluated. It was observed that there were a group of patients with a peak at grade III lesions; this suggests a slow and step by step progress of glomerular lesion in diabetics.

c. Renal vein obstruction

It is a well known fact that renal vein obstruction can cause nephrotic syndrome. According to our questionaire, only 2 cases among 5 renal vein thrombosis showed nephrotic syndrome. We have seen 10 cases of inferior vena cava syndrome, in all of which, obstruction occurred above the orifice of the renal vein. However, none of them showed nephrotic syndrome and only one case with plus two proteinuria showed diffuse thickening of glomerular capillary wall which resembled membranous glomerulopathy.

\section{Renal Circulation, Hypertension and Plasma Renin Activity}

Estimation of plasma renin activity using modified Skinner's method was carried out in various hypertensive and renal diseases. To eliminate the influences of $\mathrm{Na}$ intake and postural factor, blood samples were drawn after 7 days of $250 \mathrm{mEq} . /$ day Na-diet and early in the morning before getting up.

Plasma renin activity in various hypertensive and/or renal diseases: There was no significant difference between the peripheral plasma renin activities (PPRA) of normal and those of patients with essential hypertension.

Those with main renal artery stenosis showed high PPRA and in unilateral stenosis, ratio of plasma renin activity from each renal vein was greater than 1.5-2.0. However, 2 cases of renal artery aneurysm did not show high plasma renin activity in peripheral or renal vein blood. This fact means that renovascular hypertension 
is not always explained by increased secretion of renin. Malignant hypertension or hypertension entering a malignant phase showed high PPRA probably due to severe and diffuse vascular lesions (lamination of small arteries and fibrinoid necrosis of arterioles) acting like a stenosis in the main renal artery.

PPRA in 9 cases with chronic glomerulonephritis in its latent stage was within. normal range and most cases with hypertension in the renal failure stage did not have high PPRA, either. High PPRA is probably not responsible for high blood. pressure in these cases, but other factors such as $\mathrm{Na}$ and water retention might be suspected. Several cases of terminal renal failure showed an increase in PPRA after peritoneal dialysis, suggesting that some highly diseased kidneys are able to. secrete renin. Significantly high PPRA in nephrotic patients was observed, which. could be partly due to restriction of salt in the patient's diet.

Primary aldosteronism had very low PPRA, which was not affected by salt restriction ( $30 \mathrm{mEq} /$ day, for 4 days) or postural change ( 4 hours walking).

Regulatory mechanism of renin secretion and release: In order to clarify this. problem, an experiment was carried out in dogs. Unilateral kidneys were denervated 6 to 7 months prior to the experiment in the experimental group (the other kidneys were left intact). PAH and creatinine clearances, urine volume and electrolyte excretion and amount of secreted renin were estimated in the experimental denervated kidneys and the control kidneys in other dogs before and after aortic constriction just above the renal artery. The perfusion pressure to the kidney was measured in the femoral artery.

The same degree of decrease in perfusion pressure to the kidney caused similar changes in RPF and urine volume both in control and denervated kidneys, while in control kidneys a marked decrease in urinary $\mathrm{Na}$ excretion $(\mathrm{p}<0.01)$ and a significantly increased renin $(p<0.01)$ were observed as compared with the denervated kidneys. The results were hardly explained by the baroreceptor theory, and since efferent impulses from the renal nerve were not changed by aortic constriction, it is also unlikely that the experiment induced an excitement of the renal nerve and, in turn, of the renin secreting cells only in the innervated control kidney. After all, denervation of the kidney indirectly influenced renin secretion through a change in urinary $\mathrm{Na}$ excretion, supporting the macula densa theory. The results in this experiment were consistent with the previously reported experiment by Ohya ${ }^{\text {7) }}$ in our department who showed that a decreased tension of the renal nerve was associated with a decreased $\mathrm{Na}$ reabsorption in the renal tubules.

\section{Renal Circulation and Prognosis}

A follow up study on 110 cases with chronic glomerulonephritis was carried out. Patients were divided into 3 groups by the degree of renal function and survival curves were drawn, which showed distinct differences among 3 groups.

More than two biopsies or a biopsy and an autopsy in 29 nephritic patients with 
mesangial change (nephrotics were excluded) were carried out with an interval of more than 9 months. Patients were divided into 3 groups in accordance with types of glomerular lesion curves. Most of the cases with grade I lesion as a main change did not show a progress of glomerular lesion, while those with a peak at grade IV had a poor prognosis. Some of the intermediate group (grade II lesion as a peak) remained unchanged, some were improved, and others progressed further. So, this is a group which needs closer clinical observations.

\section{Conclusion}

Significance of renal circulation in the field of internal medicine has been discussed from the standpoint of morphology, physiology and pathophysiology. Features of hypertensive diseases or those accompanying functional or organic disorders of the renal vasculature can be well understood.

However, it is keenly felt that the knowledge on intrarenal circulation is still poor and further progress in this field is heartily desired.

\section{References}

1) Graves, E.T.: Brit. J. Surg., 42: 132, 1954.

2) Zlábek, K.: Czeckoslov. Morfol., $5: 167,1957$. c. f. 4)

3) Eguchi, S.: Jap. J. Path., 47: 323, 1958.

4) Ljungqvist, A.: Nephron, 1:329, 1964.

5) Robertson, P. W. et al.: Amer. Heart J., $73: 296,1967$.

6) Takeuchi, J. et al. : Jap. Heart J., 3: 57, 1962.

7) Ohya, N.: J. Juzen Med. Soc., 75: 534, 1967. 